\title{
PADI1 contributes to EMT in PAAD by activating the ERK1/2-p38 signaling pathway
}

\author{
Tengfei Ji, Keqiang Ma, Liang Chen, Tiansheng Cao \\ Department of Hepatobiliary Surgery, Affiliated Huadu Hospital, Southern Medical University (People’s Hospital of Huadu District), Guangzhou, China \\ Contributions: (I) Conception and design: T Cao, T Ji; (II) Administrative support: T Cao, T Ji; (III) Provision of study materials or patients: T Ji, K \\ Ma; (IV) Collection and assembly of data: T Ji, L Chen; (V) Data analysis and interpretation: T Cao, T Ji; (VI) Manuscript writing: All authors; (VII) \\ Final approval of manuscript: All authors. \\ Correspondence to: Tiansheng Cao. Department of Hepatobiliary Surgery, Affiliated Huadu Hospital, Southern Medical University (People's Hospital \\ of Huadu District), 48 Xinhua Road, Huadu District, Guangzhou 510800, China. Email: caotiansheng2088@sina.com.
}

\begin{abstract}
Background: Peptidylarginine deiminase 1 (PADI1) has been reported to promote tumorigenesis in breast cancer. However, the functional role of PADI1 in pancreatic ductal adenocarcinoma (PAAD) has remained elusive until now.
\end{abstract}

Methods: The expression pattern of PADI1 in PAAD tissues and normal tissues was analyzed using The Cancer Genome Atlas (TCGA) dataset. A Kaplan-Meier curve analysis was performed to evaluate the prognostic value of PADI1 in PAAD patients. PADI1 was knocked down in CFPAN-1 and HPAC cells, and overexpressed in PANC-1 and Bxpc-3 cells by RNA interference. A wound-healing assay was performed to analyze relative cell migration distance. Cell migration and invasion were assessed by a Transwell assay. Related protein expression levels were measured by western blot and immunofluorescence.

Results: The bioinformatics analysis showed that PADI1 was overexpressed in PAAD tissues and associated with a poor survival prognosis. The knockdown of PADI1 suppressed cell migration and invasion, and activated the ERK1/2-p38 signaling pathway in CFPAN-1 and HPAC cells. The overexpression of PADI1 produced the opposite results in PANC-1 and Bxpc-3 cells. Additionally, treatment with an MEK1/2 inhibitor significantly attenuated the effects of PADI1 knockdown on cell migration, invasion, the epithelialmesenchymal transition (EMT) process, and p-ERK1/2 and p38 expression in CFPAN-1 and HPAC cells.

Conclusions: Our data suggested that PADI1 may function as an oncogene in regulating metastasis in vitro in PAAD.

Keywords: Pancreatic ductal adenocarcinoma (PAAD); peptidylarginine deiminase 1 (PADI1); epithelialmesenchymal transition process (EMT process); ERK1/2-p38 signaling

Submitted Apr 23, 2021. Accepted for publication Jun 11, 2021.

doi: 10.21037/jgo-21-283

View this article at: https://dx.doi.org/10.21037/jgo-21-283

\section{Introduction}

Pancreatic ductal adenocarcinoma (PAAD), which accounts for nearly $85 \%$ of pancreatic cancers, represents one of the leading causes of digestive tract malignancy-related deaths, and has a 5 -year survival rate of less than $10 \%(1,2)$. Despite great progress being made in traditional therapeutic treatments, including surgical resection, chemotherapy and radiotherapy, against PAAD, the prognosis of patients with PAAD still remains poor, which can mainly be attributed distant metastasis and local invasion $(3,4)$. Various targeted therapies are being studied in clinical trials to target tumor DNA repair mechanism, tumor metabolic pathways, focal adhesion kinase, and connective tissue growth factors (5). Thus, it is of great importance to explore the underlying molecular mechanisms to improve the prognosis of this deadly disease.

Metastasis has been widely considered the major challenge in the clinical therapy of PAAD, which begins 
with the epithelial-mesenchymal transition (EMT), including the loss of cell-cell adhesion, unrestrained cell migration, and invasion $(6,7)$. The activation of the EMT is associated with the changed expression of many genes, including the downregulation of epithelial markers (e.g., occluden-1 and E-cadherin) and the upregulation of mesenchymal markers (e.g., N-cadherin and vimentin) (8). In addition, the mitogen-activated protein kinase (MAPK) pathway plays a significant role in the initiation of the EMT, which is prevalent in many cancers, including pancreatic cancer (9) and ovarian cancer (10). The MAPK family includes three subfamilies (i.e., ERK1/2, p38, and JNK), all of which can be triggered by growth or stress factors, and thus play a vital part in signal transduction inside cells $(10,11)$. Therefore, ERK1/2 and p38 appear to be closely correlated to the EMT process in PAAD cells.

The peptidylarginine deiminase (PAD) family comprises five members (i.e., PADI1-4, and 6) that are located within a highly organized gene cluster at $1 \mathrm{p} 36.13$ in humans and on the orthologous region of mouse chromosome 4, and study has found that PAD could post-translationally convert arginine residues into neutrally charged citrulline (12). Interestingly, recent studies have reported on the important role of PADI-mediated protein citrullination in various cancers (13). For example, Stadler et al. (14) reported that PADI4 overexpression inhibited breast cancer cell migration via its role in modulating EMT. While Xin et al. (15) reported that silencing PADI4 suppressed cell proliferation, cell cycle progression, and invasion in gastric cancer cells. PADI2 has been identified as a potential breast cancer biomarker and therapeutic target (16). The inhibition of PADI2 could enhance the anti-tumor effect of docetaxel in tamoxifen-resistant breast cancer cells (17). A recent report by Qin et al. (18) not only showed that PADI1 depletion suppressed tumorigenesis in triple negative breast cancer, but that these changes were correlated with the activated ERK1/2 and p38 MAPK signaling pathways. However, whether PADI1 regulates metastasis and the EMT in PAAD by modulating ERK1/2-p38 signaling pathway remains unclear.

In the present study, we first investigated the expression pattern and survival prognosis of PADI1 in PAAD patients. We then performed loss-of-function and gain-of-function assays to analyze the effects of PADI1 on PAAD cell migration, invasion, the EMT, and ERK1/2-p38 signaling. Additionally, rescue experiments were performed to determine whether PADI1 regulated PAAD cell migration and invasion by regulating the ERK1/2-p38 signaling pathway. We present the following article in accordance with the MDAR reporting checklist (available at https:// dx.doi.org/10.21037/jgo-21-283).

\section{Methods}

\section{Database analysis}

The messenger RNA (mRNA) expression pattern of PADI1 in primary PAAD tissues and normal tissues examined in this study is available in the public repository of The Cancer Genome Atlas (TCGA; https://portal.gdc.cancer. gov/) database. Based on the survival data of PAAD patients obtained from TCGA database, we investigated the effect of PADI1 expression levels on PAAD patient survival rate using the Kaplan-Meier method with a log-rank test. The study was conducted in accordance with the Declaration of Helsinki (as revised in 2013).

\section{Cell lines and treatments}

A total of five PAAD cell lines (i.e., CFPAC-1, PANC-1, PL45, Bxpc-3, and HPAC) and human pancreatic nestinexpressing cells (hTERT-HPNE) were obtained from the Cell Resource Center of Shanghai Institute of Life Sciences, Chinese Academy of Sciences. All cell lines were cultured in the recommended conditions, and supplemented with $10 \%$ fetal bovine serum (FBS, Hyclone Co., Logan, UT, USA) in a humidified $5 \%$ carbon dioxide $\left(\mathrm{CO}_{2}\right)$ air incubator at $37^{\circ} \mathrm{C}$.

The small interfering RNA targeting PADI1 (siPADI1), the PADI1 mimics overexpression plasmid, and the corresponding controls were synthesized by Shanghai Genepharma, Co., Ltd. (Shanghai, China). For cell transfection, CFPAC-1, PANC-1, Bxpc-3, and HPAC cells were seeded into 6 -well plates at a density of $1 \times 10^{7}$ cells per well. The CFPAC-1 and HPAC cells were transfected with siPADI1 or siNC as the control, while PANC-1 and Bxpc-3 cells were transfected with PADI1 mimics or an empty vector in accordance with the instructions provided by Lipofectamine 3000 (Life Technology, MD, USA) for $48 \mathrm{~h}$. For the validated experiments, CFPAC- 1 and HPAC cells were treated with an ERK1/2 inhibitor (compound 27, Sigma-Aldrich, St. Louis, MO, USA) for $2 \mathrm{~h}$, followed by siPADI1 transfection.

\section{Quantitative real-time polymerase chain reaction (PCR)}

The total RNA from the cell lines was isolated with a 
TRIzol reagent (Invitrogen, Carlsbad, CA, USA) and complementary DNA (cDNA) was synthesized from $2 \mu \mathrm{g}$ of RNA with the PrimeScript ${ }^{\mathrm{TM}}$ RT Reagent Kit (Takara Biotechnology, Dalian, China) in accordance with the manufacturer's protocols. A quantitative realtime PCR was performed on the ABI StepOnePlus Realtime PCR system with SYBR Premix Ex Taq ${ }^{\text {TM }}$ (Takara, Dalian, China) according to the following cycling conditions: $95^{\circ} \mathrm{C}$ for $30 \mathrm{sec}$, followed by 40 cycles of $95^{\circ} \mathrm{C}$ for $5 \mathrm{sec}$, and $60{ }^{\circ} \mathrm{C}$ for $34 \mathrm{sec}$. The primer sequences used in this study were as follows: PADI1 forward: 5'-TTTCGGTGCTTTGAGTGGGTC-3' and reverse: 5'-TGTTGGCATATTTGTGGCAGG-3'; GAPDH forward: 5'-CACCCACTCCTCCACCTTTG-3' and reverse: 5'-CCACCACCCTGTTGCTGTAG-3'. The reactions were performed in triplicate, and relative fold changes in PADI1 mRNA expression were calculated using the $2^{-\Delta \Delta C T}$ method.

\section{Western-blot analysis}

A total protein sample was extracted from the cell lines using a radioimmunoprecipitation assay (RIPA) buffer (Beyotime, Shanghai, China) and protein concentration was determined using a bicinchoninic acid protein assay kit (Beyotime). An equal amount of protein sample was separated by $10 \%$ sodium dodecyl sulfate polyacrylamide gel electrophoresis and transferred onto polyvinylidene fluoride membranes (Millipore). The membranes were blocked with $5 \%$ non-fat milk for $2 \mathrm{~h}$ and incubated with primary antibodies against PADI1, E-cadherin, $\mathrm{N}$-cadherin, Vimentin, p-ERK1/2, p38, p-p38, and GAPDH (all dilution: 1:1,000, Abcam, UK), after which they were incubated with a horseradish peroxidase-conjugated secondary antibody. The immunoreactive bands were then analyzed with an electrochemiluminescence system (GE Healthcare).

\section{Immunofluorescence}

Transfected cells from different groups were seeded into chamber slides and cultured overnight. The next day, the cells were fixed with $4 \%$ formaldehyde for $30 \mathrm{~min}$ and permeabilized with $0.5 \%$ Triton X-100. After being blocked with $1 \%$ bovine serum albumin for $30 \mathrm{~min}$, the cells were incubated with PADI1 and p-ERK1/2 antibodies overnight at $4{ }^{\circ} \mathrm{C}$. The cells were then washed with phosphatebuffered saline (PBS) and incubated with an appropriated secondary antibody. The nuclei were counterstained with 4',6-diamidino-2-phenylindole (DAPI, Sigma). The stained images were observed and analyzed via immunofluorescence microscopy.

\section{Wound-healing assay}

In brief, the transfected cells were plated into 24-well plates and grown to 100 confluence. Afterwards, the cells from different groups were scratched with a $10 \mu \mathrm{L}$ pipette tip (time $0)$. Next, the cells were washed with PBS to remove isolated cells, which were further incubated with a complete growth medium. After $24 \mathrm{~h}$, images of cells that had migrated to the injured area were obtained using an inverted microscope.

\section{Transwell assay}

Cell migration and invasion ability were assessed by a $24-w e l l$ Transwell chamber precoated without and with $1 \mathrm{mg} / \mathrm{mL}$ Matrigel (Millipore, MA, USA), respectively. Briefly, approximately $2.0 \times 10^{4}$ transfected cells from different groups suspended in $100 \mu \mathrm{L}$ of serum-free medium were inoculated in the upper chamber, and $500 \mu \mathrm{L}$ of complete medium was added to the lower chamber as the chemoattractant. After $48 \mathrm{~h}$, the cells that migrated into the lower chamber were fixed with $75 \%$ ethanol, stained with $0.5 \%$ crystal violet, and counted under a light microscope (BD Biosciences, Heidelberg, Germany).

\section{Statistical analysis}

The statistical data were analyzed with GraphPad prism 6 and expressed as means \pm standard deviations (SDs) from three independent experiments. The paired Student's $t$-test was applied to determine the statistical significance of the differences between the two groups. A one-way analysis of variance, followed by Dunnett's test was used to assess the statistical significance of differences between multiple groups. Differences were considered statistically significant at $\mathrm{P}<0.05$.

\section{Results}

\section{PADI1 was overexpressed in PAAD tissues and associated with a poor survival prognosis}

To elucidate the role of PADI1 in PAAD pathogenesis, we first searched the expression pattern of PADI1 in primary 


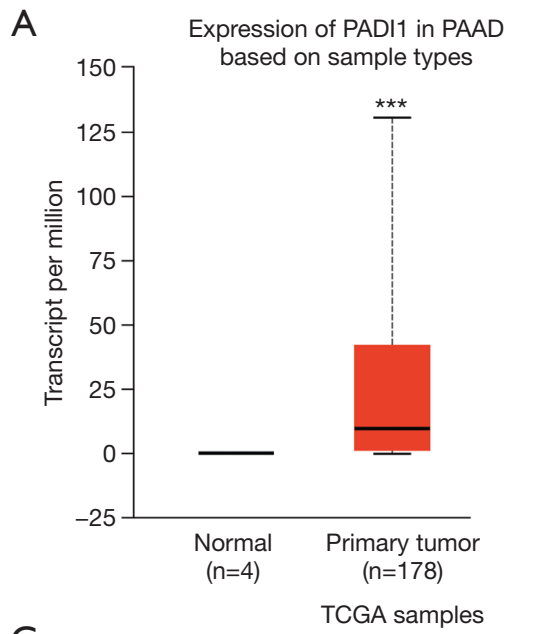

C

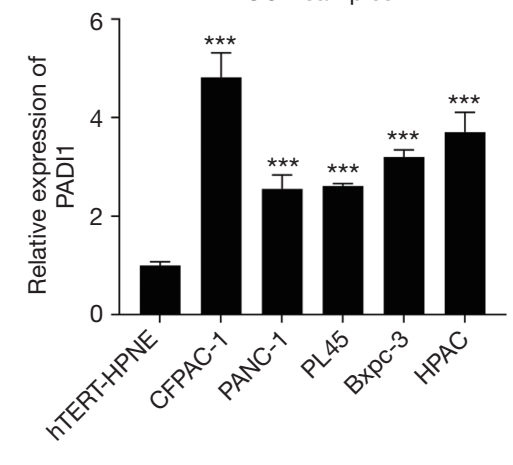

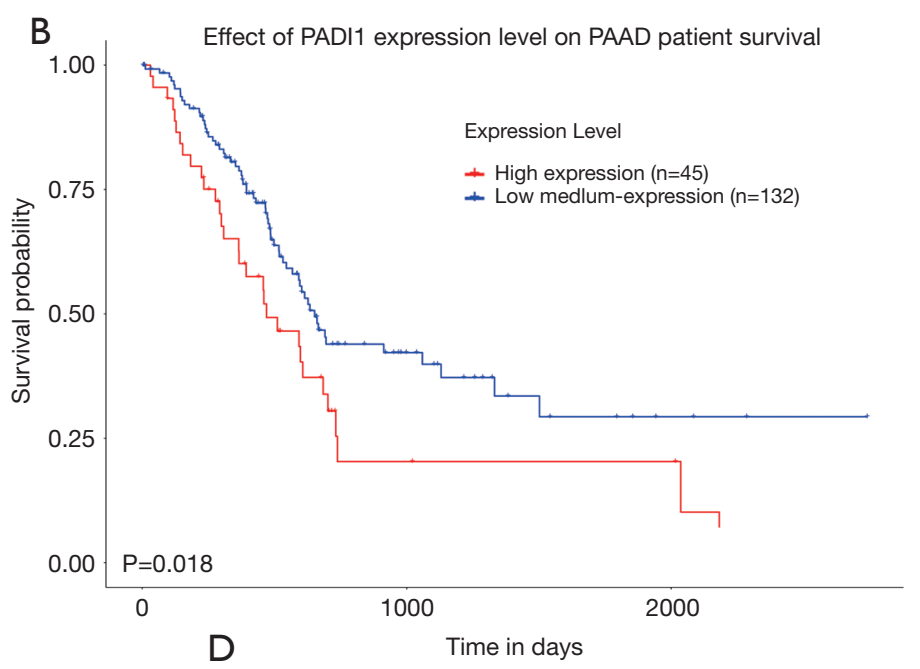
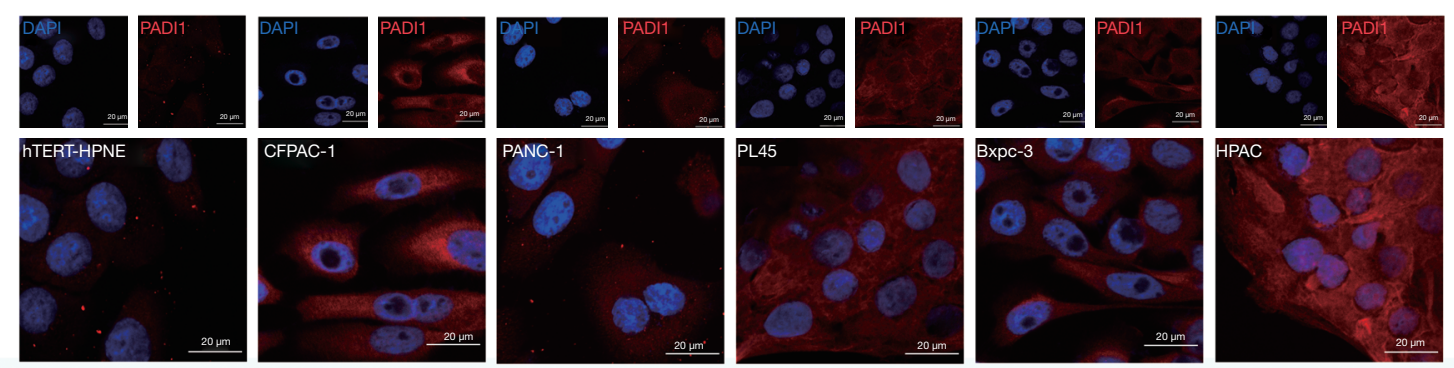

Figure 1 The expression of PADI1 and prognosis in PAAD patients. TCGA database was searched to investigate (A) the expression of PADI1 in tumor and normal tissues, and (B) the effects of PADI1 on the survival prognosis of PAAD patients. ${ }^{* * *}, \mathrm{P}<0.001$, compared with normal. (C) A quantitative real-time PCR and (D) western-blot analysis were applied to determine the expression of PADI1 mRNA and protein levels, respectively, in five PAAD cell lines (i.e., CFPAC-1, PANC-1, PL45, Bxpc-3, and HPAC) and the human pancreatic cell line, hTERT-HPNE. ***, $\mathrm{P}<0.001$, compared with the hTERT-HPNE. (E) PADI1 was observed using immunofluorescence (400×) in PAAD cell lines and hTERT-HPNE. PADI1, peptidylarginine deiminase 1; PAAD, pancreatic ductal adenocarcinoma; TCGA, The Cancer Genome Atlas; PCR, polymerase chain reaction; mRNA, messenger RNA.

tumor and normal tissues derived from PAAD patients on TCGA database. As Figure $1 A$ shows, PADI1 was found to be significantly overexpressed in PAAD tumor samples $(n=178)$ compared to normal tissues $(n=4)$. An analysis of TCGA datasets indicated that high PADI1 expression levels in PAAD samples were associated with a poor survival prognosis (see Figure $1 B$ ). To further validate the expression of PADI1, we performed a quantitative real-time PCR and western-blot analysis to determine the expression of PADI1 in PAAD cell lines. The results showed that the expression of PADI1 mRNA (see Figure 1C) and protein (see Figure 1D) was obviously 
upregulated in PAAD cell lines, including CFPAC-1, PANC-1, PL45, Bxpc-3, and HPAC, compared with the normal pancreatic cell line, hTERTHPNE. Similarly, immunofluorescence staining of PADI1 confirmed the overexpression of PADI1 in PAAD cell lines compared with hTERT-HPNE cells (see Figure 1E).

\section{The knockdown of PADI1 suppressed PAAD cell migration and invasion, and activated the ERK1/2-p38 signaling pathway}

To determine the functional role of PADI1 in PAAD cells, we first performed loss-of-function assays in CFPAN-1 and HPAC cells with relatively high PADI1 expression by transfection with siPADI1. As the PCR (see Figure $2 A$ ) and western-blot analysis (see Figure $2 B$ ) showed, the expression of PADI1 was significantly decreased in CFPAC-1 and HPAC cells after siPADI1 transfection compared to that of the control group. The results of the wound-healing assay indicated that the knockdown of PADI1 obviously suppressed the healing rate of CFPAN-1 and HPAC cells (see Figure 2C). The Transwell assay further demonstrated that the knockdown of PADI1 significantly suppressed the migration (see Figure 2D,E) and invasion (see Figure 2F,G) ability of both CFPAN-1 and HPAC cells. At the molecular level, the knockdown of PADI1 suppressed the EMT progress, as reflected by elevated E-cadherin expression and reduced $\mathrm{N}$-cadherin and Vimentin expression, and upregulated p-ERK1/2 and p-38 expression in CFPAN-1 and HPAC cells (see Figure 2H). The immunofluorescence assay further confirmed the protein expression of p-ERK1/2 was increased after PADI1 knockdown in CFPAN-1 and HPAC cells (see Figure 2I).

\section{The overexpression of PADI1 promoted PAAD cell migration, invasion and the ERK1/2-p38 signaling patbway}

To further confirm the oncogenic role of PADI1 in PAAD cells, we selected PANC-1 and Bxpc-3 cells with relatively low PADI1 expression to transfect with the PADI1 overexpression plasmid. As Figure $3 A, B$ shows, the expression of PADI1 was remarkedly upregulated after PADI1 mimics plasmid transfection compared to that of the empty vector in PANC-1 and Bxpc-3 cells. In contrast to PADI1 knockdown, we observed that the overexpression of PADI1 notably promoted the healing rate (see Figure 3C), cell migration (see
Figure 3D,E) and invasion (see Figure 3F,G) ability in PANC1 and Bxpc-3 cells. Similarly, PADI1 overexpression produced the opposite effects on the protein levels of E-cadherin, $\mathrm{N}$-cadherin, Vimentin, p-ERK1/2, p38, and p-p38, as depicted by the western-blot analysis (see Figure $3 H$ ) and immunofluorescence assay (see Figure 3I).

\section{PADI1 affected the EMT process by regulating the ERK1/2-p38 signaling patbway in PAAD cells}

Next, we examined whether PADI1 regulated PAAD cell migration and invasion by regulating the ERK1/2-p38 signaling pathway. The CFPAC- 1 and HPAC cells were first transfected with siPADI1 and then treated with an ERK1/2 inhibitor (compound 27). As depicted by the wound-healing assay, the inhibition of p-ERK1/2 obviously promoted the cell healing rate in PADI1 silenced CFPAC-1 and HPAC cells (see Figure 4A). Similarly, the cell migration (see Figure 4B,C) and invasion (see Figure $4 D, E$ ) ability were enhanced in PADI1 silenced CFPAC-1 and HPAC cells after p-ERK1/2 inhibition. Further, we found that the inhibition of p-ERK1/2 in PADI1 silenced CFPAC-1, and HPAC cells activated the EMT process and suppressed the ERK1/2-p38 signaling pathway (see Figure 4F,G).

\section{Discussion}

We first observed that the expression of PADI1 was upregulated in PAAD tissues compared to normal tissues, and correlated with a poor survival prognosis in the TCGA database. It has been reported that the PADI enzyme family is linked to carcinogenesis and tumor progression $(16,19,20)$. Similar to our findings, Qin et al. (18) searched the Oncomine and TCGA databases and found that the expression of PADI1 was upregulated in breast cancer patients, and positively correlated with triple negative breast cancer. Wang et al. (21) showed that PADI2, an androgen-repressed gene, is upregulated in castration-resistance prostate cancer. Liu et al. (22) found that PADI4 was overexpressed in lung cancer tissues and cell lines. Based on these findings, we speculated that PADI1 might be involved in regulating PAAD progression. A latest research found that PADI1 is up-regulated in a variety of cancer cell types (23), including melanoma, breast cancer, renal cell carcinoma and cervical carcinoma, which also proved PADI1 plays an important role in cancer cells.

We then investigated the functional role of PADI1 in 
A

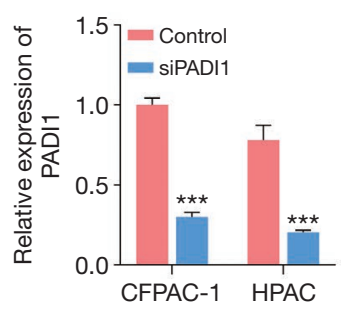

C

$\mathrm{Oh}$

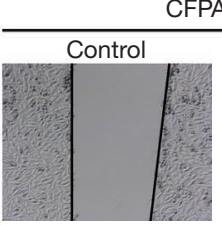

$24 \mathrm{~h}$

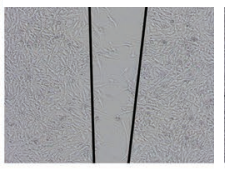

E

D

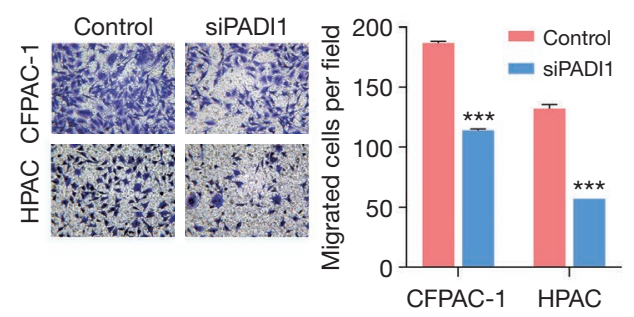

B
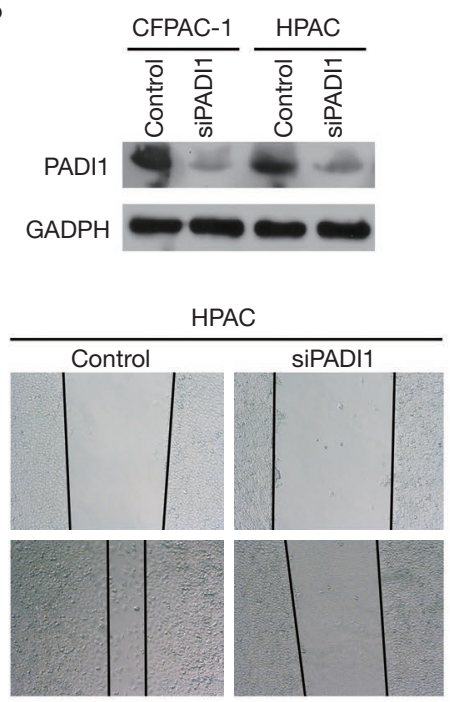

F

G

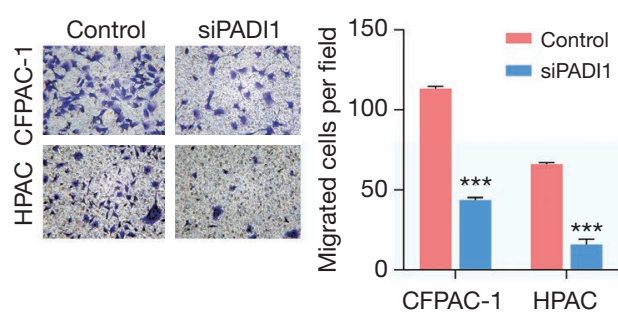

Control
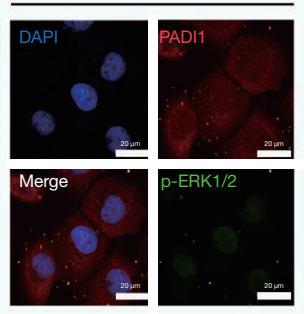

Merge

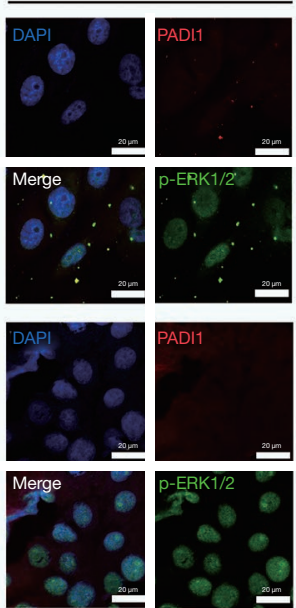

SiPADI1

Figure 2 The knockdown of PADI1 suppressed PAAD cell migration and invasion, and activated the ERK1/2-p38 signaling pathway. CFPAC-1 and HPAC cells were transfected with siPADI1 or a control. (A) A quantitative real-time PCR and (B) western-blot analysis were performed to determine the expression of PADI1 mRNA and protein levels in the above two cell lines. (C) A wound-healing assay was performed to assess cell migration in the above two cell lines (40x). A Transwell assay was used to evaluate cell migration (D,E) and invasion $(\mathrm{F}, \mathrm{G})$ in the above two cell lines $(100 \times$, stained with crystal violet). Data are expressed as means \pm SD from three independent experiments. ***, $\mathrm{P}<0.001$, compared with the control. (H) The protein expression of E-cadherin, N-cadherin, Vimentin, p-ERK1/2, p38, and p-p38 was measured in the above two cell lines by a western-blot analysis. (I) PADI1 and p-ERK1/2 expression levels were observed using immunofluorescence (400x) in the above two cell lines. PADI1, peptidylarginine deiminase 1; PAAD, pancreatic ductal adenocarcinoma; PCR, polymerase chain reaction; mRNA, messenger RNA; SD, standard deviation. 
A

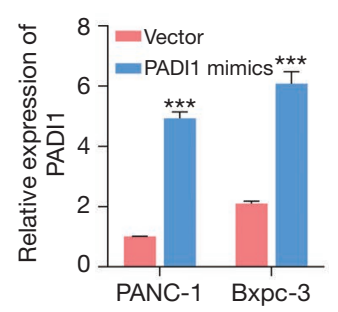

C

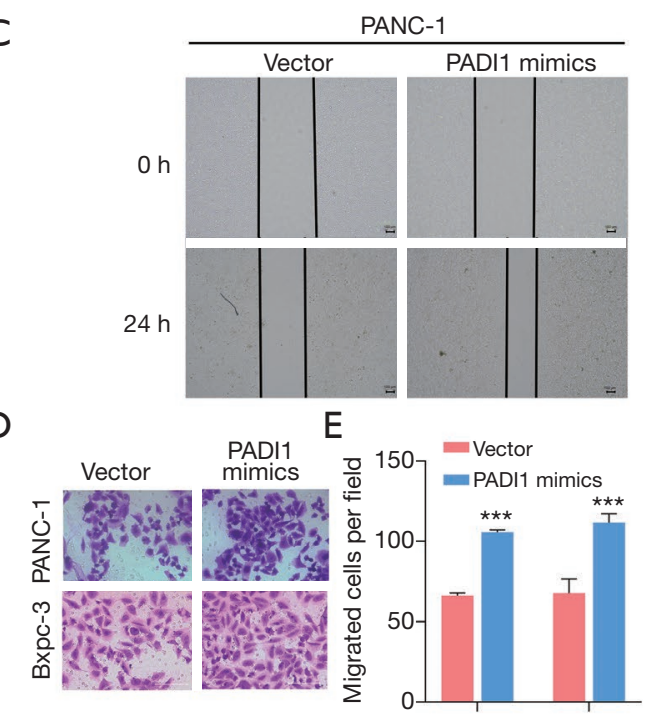

B

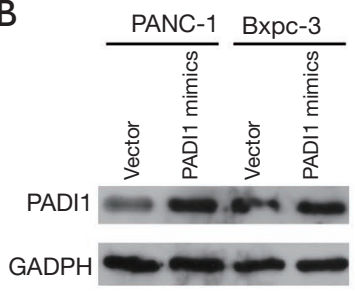

Bxpc-3

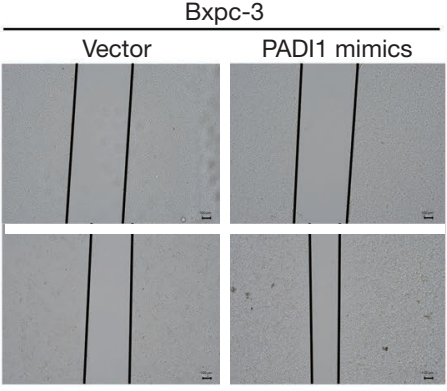

$\mathrm{F}$

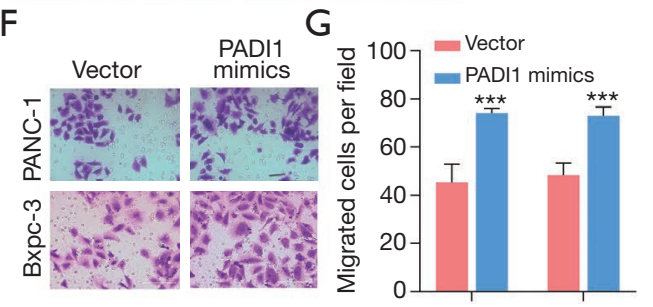

$\mathrm{H}$

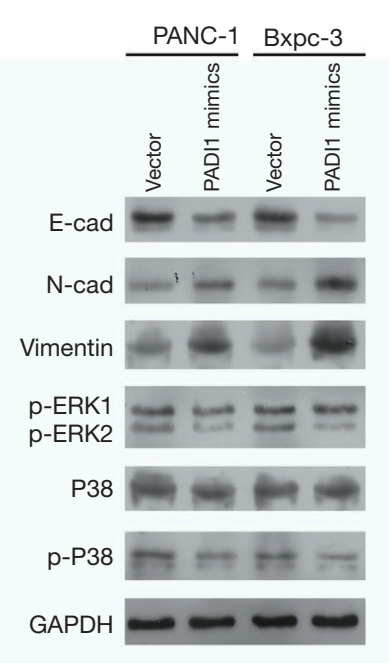

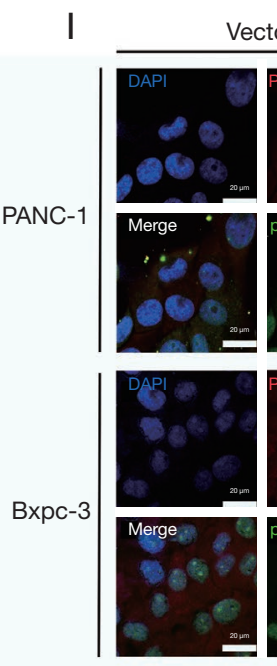

Vector
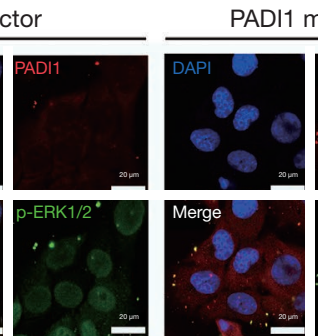

ADI1 mimics

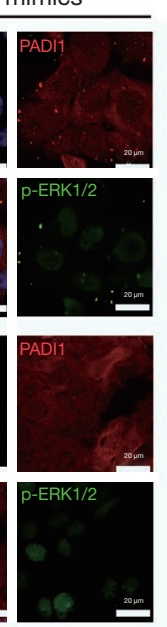

Figure 3 The overexpression of PADI1 promoted PAAD cell migration, invasion, and the ERK1/2-p38 signaling pathway. PANC-1 and Bxpc-3 cells were transfected with the PADI1 mimics overexpression plasmid or vector. (A) Quantitative real-time PCR and (B) westernblot analysis were applied to determine the expression of PADI1 mRNA and protein levels in the above two cell lines. (C) A wound-healing assay was performed to assess cell migration in the above two cell lines (40x). Transwell assay was used to evaluate cell migration (D,E) and invasion $(\mathrm{F}, \mathrm{G})$ in the above two cell lines (100x, stained with crystal violet). Data are expressed as means \pm SD from three independent experiments. ${ }^{* * *}, \mathrm{P}<0.001$, compared with vector. $(\mathrm{H})$ The protein expression of E-cadherin, N-cadherin, Vimentin, p-ERK1/2, p38, and p-p38 was measured in the above two cell lines by a western-blot analysis. (I) PADI1 and p-ERK1/2 expression levels were observed using immunofluorescence $(400 x)$ in the above two cell lines. PADI1, peptidylarginine deiminase 1; PAAD, pancreatic ductal adenocarcinoma; PCR, polymerase chain reaction; mRNA, messenger RNA; SD, standard deviation. 
A

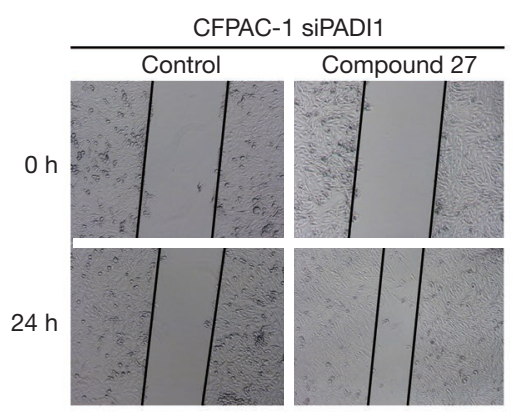

B

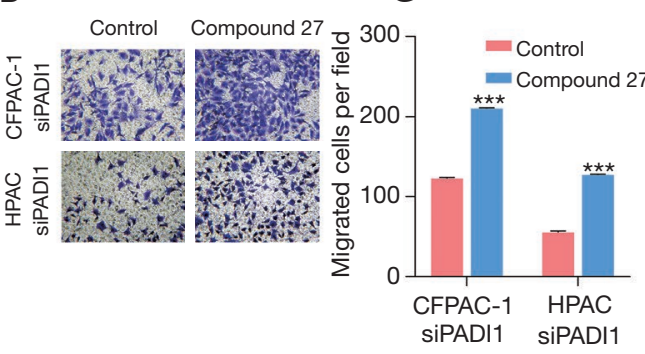

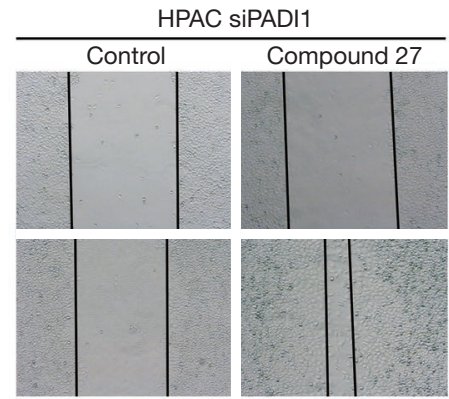

$\mathrm{E}$

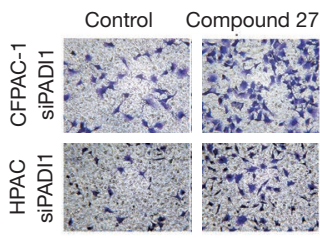

$\mathrm{F}$

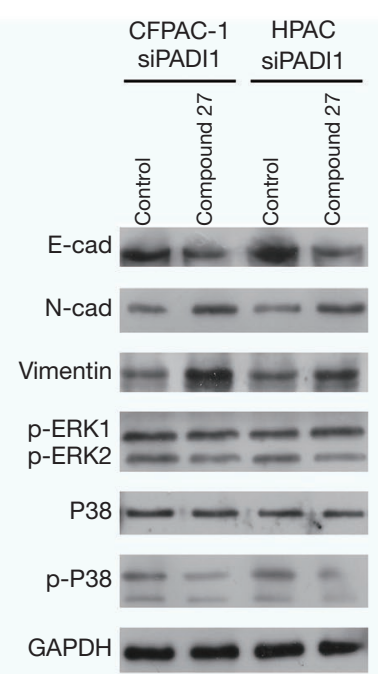

G
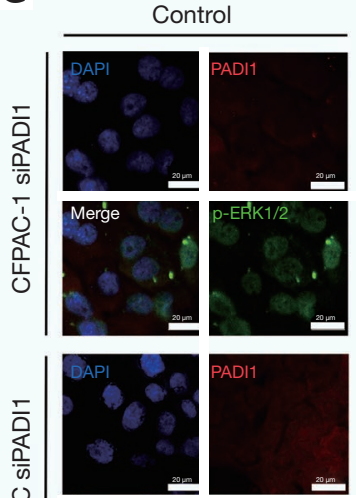

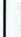

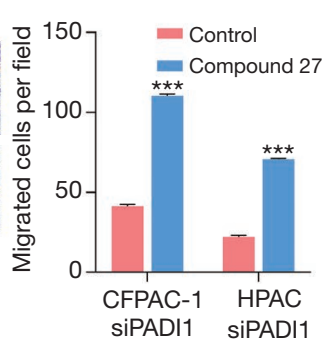

Figure 4 PADI1 affected the EMT process by regulating the ERK1/2-p38 signaling pathway in PAAD cells. The CFPAC-1 and HPAC cells were first treated with an ERK1/2 inhibitor (compound 27) and then transfected with siPADI1. (A) A wound-healing assay was performed to assess cell migration in the above two cell lines (40x). A Transwell assay was used to evaluate cell migration $(B, C)$ and invasion $(D, E)$ in the above two cell lines $\left(100 \times\right.$, stained with crystal violet). Data are expressed as means $\pm \mathrm{SD}$ from three independent experiments. ${ }^{* * *}, \mathrm{P}<0.001$, compared with the control. (F) The protein expression of E-cadherin, N-cadherin, Vimentin, p-ERK1/2, p38, and p-p38 was measured in the above two cell lines by a western-blot analysis. (G) PADI1 and p-ERK1/2 expression levels were observed using immunofluorescence $(400 \times)$ in the above two cell lines. PADI1, peptidylarginine deiminase 1; EMT, epithelial-mesenchymal transition; PAAD, pancreatic ductal adenocarcinoma; SD, standard deviation. 
PAAD in vitro. As expected, PADI1 knockdown suppressed the migration and invasion ability of PAAD cells, while PADI1 overexpression produced the opposite results. Our in vitro data suggested that PADI1 exerted oncogenic effects on metastasis in PAAD cells. At the molecular level, we observed that the knockdown of PADI1 elevated the expression of E-cadherin, p-ERK1/2, and p-38, and reduced $\mathrm{N}$-cadherin and Vimentin expression in CFPAN-1 and HPAC cells. Conversely, PADI1 overexpression produced the opposite results on the above protein expression levels in PANC-1 and Bxpc-3 cells. ERK and p38 are two major MAPKs that have been extensively studied in mammals (24). In the present study, we showed that both p-ERK1/2 and p-38 were activated in PADI1 depleted cells. Thus, PADI1appears to have an inhibitory effect on the activation of ERK1/2-p38 signaling. Consistent with our data, PADI1 silencing reduced cell proliferation, suppressed the EMT, prevented metastasis, and activated the ERK1/2 and p38 MAPK signaling pathways in MDA-MB-231 cells (18). PADI4 promoted the EMT while the PADI4 inhibitor suppressed the EMT in osteosarcoma cells (25). Downregulating PADI2 suppressed the colony formation, proliferation, migration, and invasion of A2780 and SKOV3 cells (26). Conversely, the overexpression of PADI4 constrained the activity of EMT by suppressing ETS-domain containing protein (Elk1) expression, and inhibited the resistance of non-small cell lung cancer to gefitinib (27). From these reports, the different effects of PADIs in different tumor cells might mainly be ascribed to different tissue sources.

Further, we found evidence that ERK1/2 and p38 play distinct roles in the migration, invasion and EMT process in PADI1 depleted CFPAC-1 and HPAC cells. Our data described the activated migration, invasion, and EMT process following the inhibition of p-ERK1/2 in PADI1 silenced CFPAC-1 and HPAC cells, which indicated that ERK1/2 inactivation appears to be primarily responsible for the upregulation of $\mathrm{N}$-cadherin and Vimentin, and the downregulation of E-cadherin expression in PADI1 knockdown cells. Currently, the precise role of MAPK signaling in regulating migration, invasion, and the EMT remains controversial. The epidermal growth factor downregulates E-cadherin expression via the production of hydrogen peroxide, the activation of $\mathrm{p} 38 \mathrm{MAPK}$, and the upregulation of SNAIL in human ovarian cancer cells (28). Pirfenidone is able to attenuate the EMT and fibrosis in vivo and in vitro by antagonizing the MAPK pathway (29). Different cell types are no doubt the major reasons for these controversies.
Taken together, PADI1 exerted oncogenic effects on the PAAD cells in vitro by promoting cell migration and invasion and the EMT process, all of which might be associated with the inactivation of the ERK1/2-p38 signaling pathway. Our research indicates that targeting PADI1 in PAAD may represent a promising treatment.

\section{Acknowledgments}

Funding: This research was funded by grants from The Science and Technology Program of Guangzhou (No. 201904010179).

\section{Footnote}

Reporting Checklist: The authors have completed the MDAR reporting checklist. Available at https://dx.doi. org/10.21037/jgo-21-283

Data Sharing Statement: Available at https://dx.doi. org/10.21037/jgo-21-283

Conflicts of Interest: All authors have completed the ICMJE uniform disclosure form (available at https://dx.doi. org/10.21037/jgo-21-283). The authors have no conflicts of interest to declare.

Etbical Statement: The authors are accountable for all aspects of the work in ensuring that questions related to the accuracy or integrity of any part of the work are appropriately investigated and resolved. The study was conducted in accordance with the Declaration of Helsinki (as revised in 2013).

Open Access Statement: This is an Open Access article distributed in accordance with the Creative Commons Attribution-NonCommercial-NoDerivs 4.0 International License (CC BY-NC-ND 4.0), which permits the noncommercial replication and distribution of the article with the strict proviso that no changes or edits are made and the original work is properly cited (including links to both the formal publication through the relevant DOI and the license). See: https://creativecommons.org/licenses/by-nc-nd/4.0/.

\section{References}

1. Ilic M, Ilic I. Epidemiology of pancreatic cancer. World J 
Gastroenterol 2016;22:9694-705.

2. Siegel RL, Miller KD, Jemal A. Cancer statistics, 2018. CA Cancer J Clin 2018;68:7-30.

3. Rhim AD, Mirek ET, Aiello NM, et al. EMT and dissemination precede pancreatic tumor formation. Cell 2012;148:349-61.

4. Nielsen SR, Quaranta V, Linford A, et al. Macrophagesecreted granulin supports pancreatic cancer metastasis by inducing liver fibrosis. Nat Cell Biol 2016;18:549-60. Erratum in: Nat Cell Biol 2016;18:822.

5. Chi J, Chung SY, Parakrama R, et al. The role of PARP inhibitors in BRCA mutated pancreatic cancer. Therap Adv Gastroenterol 2021;14:17562848211014818.

6. Nakajima S, Doi R, Toyoda E, et al. N-cadherin expression and epithelial-mesenchymal transition in pancreatic carcinoma. Clin Cancer Res 2004;10:4125-33.

7. Yilmaz M, Christofori G, Lehembre F. Distinct mechanisms of tumor invasion and metastasis. Trends Mol Med 2007;13:535-41.

8. Seton-Rogers S. Epithelial-mesenchymal transition: untangling EMT's functions. Nat Rev Cancer 2016;16:1.

9. Sheng $W$, Chen C, Dong $M$, et al. Calreticulin promotes EGF-induced EMT in pancreatic cancer cells via Integrin/ EGFR-ERK/MAPK signaling pathway. Cell Death Dis 2017;8:e3147.

10. Zhu J, Zheng Y, Zhang H, et al. Galectin-1 induces metastasis and epithelial-mesenchymal transition (EMT) in human ovarian cancer cells via activation of the MAPK JNK/p38 signalling pathway. Am J Transl Res 2019;11:3862-78.

11. Miao JH, Wang SQ, Zhang MH, et al. Knockdown of galectin-1 suppresses the growth and invasion of osteosarcoma cells through inhibition of the MAPK/ERK pathway. Oncol Rep 2014;32:1497-504.

12. Vossenaar ER, Zendman AJ, van Venrooij WJ, et al. PAD, a growing family of citrullinating enzymes: genes, features and involvement in disease. Bioessays 2003;25:1106-18.

13. Chang X, Han J. Expression of peptidylarginine deiminase type 4 (PAD4) in various tumors. Mol Carcinog 2006;45:183-96.

14. Stadler SC, Vincent CT, Fedorov VD, et al. Dysregulation of PAD4-mediated citrullination of nuclear GSK3 $\beta$ activates TGF- $\beta$ signaling and induces epithelial-tomesenchymal transition in breast cancer cells. Proc Natl Acad Sci U S A 2013;110:11851-6. Proc Natl Acad Sci U S A $2013 ; 110: 16283$.
15. Xin J, Song X. Role of peptidylarginine deiminase type 4 in gastric cancer. Exp Ther Med 2016;12:3155-60.

16. McElwee JL, Mohanan S, Griffith OL, et al. Identification of PADI2 as a potential breast cancer biomarker and therapeutic target. BMC Cancer 2012;12:500.

17. Li F, Miao L, Xue T, et al. Inhibiting PAD2 enhances the anti-tumor effect of docetaxel in tamoxifen-resistant breast cancer cells. J Exp Clin Cancer Res 2019;38:414.

18. Qin H, Liu X, Li F, et al. PAD1 promotes epithelialmesenchymal transition and metastasis in triple-negative breast cancer cells by regulating MEK1-ERK1/2-MMP2 signaling. Cancer Lett 2017;409:30-41.

19. Wang Y, Li P, Wang S, et al. Anticancer peptidylarginine deiminase $(\mathrm{PAD})$ inhibitors regulate the autophagy flux and the mammalian target of rapamycin complex 1 activity. J Biol Chem 2012;287:25941-53.

20. Chang X, Han J, Pang L, et al. Increased PADI4 expression in blood and tissues of patients with malignant tumors. BMC Cancer 2009;9:40.

21. Wang L, Song G, Zhang X, et al. PADI2-mediated citrullination promotes prostate cancer progression. Cancer Res 2017;77:5755-68.

22. Liu M, Qu Y, Teng X, et al. PADI4-mediated epithelial-mesenchymal transition in lung cancer cells. Mol Med Rep 2019;19:3087-94.

23. Coassolo S, Davidson G, Negroni L, et al. Citrullination of pyruvate kinase $\mathrm{M} 2$ by PADI1 and PADI3 regulates glycolysis and cancer cell proliferation. Nat Commun 2021;12:1718.

24. Dhillon AS, Hagan S, Rath O, et al. MAP kinase signalling pathways in cancer. Oncogene 2007;26:3279-90.

25. Zhai Q, Qin J, Jin X, et al. PADI4 modulates the invasion and migration of osteosarcoma cells by downregulation of epithelial-mesenchymal transition. Life Sci 2020;256:117968.

26. Liu L, Zhang Z, Zhang G, et al. Down-regulation of PADI2 prevents proliferation and epithelial-mesenchymal transition in ovarian cancer through inhibiting JAK2/ STAT3 pathway in vitro and in vivo, alone or in combination with Olaparib. J Transl Med 2020;18:357.

27. Duan Q, Pang C, Chang N, et al. Overexpression of PAD4 suppresses drug resistance of NSCLC cell lines to gefitinib through inhibiting Elk1-mediated epithelial-mesenchymal transition. Oncol Rep 2016;36:551-8. 
28. Cheng JC, Klausen C, Leung PC. Hydrogen peroxide mediates EGF-induced down-regulation of E-cadherin expression via p38 MAPK and snail in human ovarian cancer cells. Mol Endocrinol 2010;24:1569-80.

29. Li Z, Liu X, Wang B, et al. Pirfenidone suppresses MAPK signalling pathway to reverse epithelial-mesenchymal transition and renal fibrosis. Nephrology (Carlton) 2017;22:589-97.

(English Language Editor: L. Huleatt)

Cite this article as: Ji T, Ma K, Chen L, Cao T. PADI1 contributes to EMT in PAAD by activating the ERK1/2-p38 signaling pathway. J Gastrointest Oncol 2021;12(3):1180-1190. doi: 10.21037/jgo-21-283 